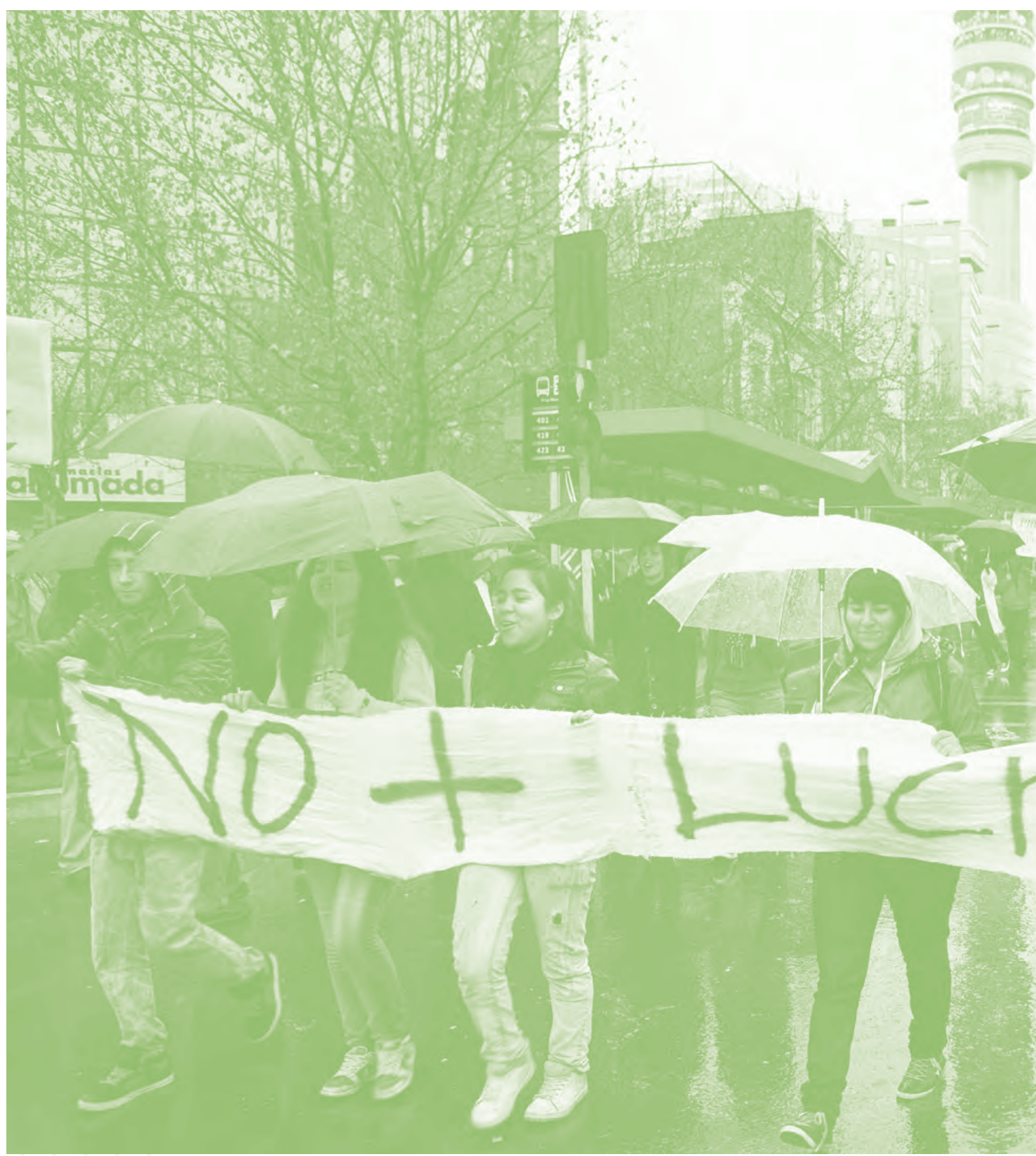


en una serie de compromisos políticos del foro internacional. ${ }^{267}$

Sin perjuicio de lo anterior, muchas lagunas normativas aún persisten respecto a su implementación, especialmente en contextos globales donde la privatización y la mercantilización de la educación han ganado espacio. Los denominados Principios de Abiyán buscan precisamente hacerse cargo de este desafío al ofrecer guías para los Estados en relación con la garantía de una educación pública, gratuita y de calidad por una parte, y la regulación de la participación del sector privado en su provisión, por otra.

En este contexto, este artículo argumenta que los Principios de Abiyán permiten hacerse cargo de las brechas constitucionales del derecho a la educación en Chile en relación con temas clave tales como el propósito de la educación, los sistemas de educación pública, el financiamiento, la libertad de elección de la escuela y la privatización de la educación. De esta forma, el proceso chileno ofrece una oportunidad institucional única para integrar por primera vez los Principios de Abiyán en un mecanismo de creación constitucional, y con ello transformar un sistema educativo marcado por la segregación y la desigualdad.

B. En 2019, con el objetivo de compilar las obligaciones internacionales

Los Principios de Abiyán y el derecho a la educación de derechos humanos en relación al derecho a la educación, 57 de los más cualificados expertos del mundo adoptaron "los Principios rectores sobre las obligaciones de derechos humanos que incumben a los Estados de proporcionar educación pública y regular la participación del sector privado en la educación", más conocidos como los Principios de Abiyán ${ }^{268}$ (en adelante también, Los Principios).

En un contexto global de creciente privatización y comercialización de la educación, en que los intereses privados y la obtención de beneficios toman más protagonismo que el respeto, la protección y la realización del derecho a la educación, ${ }^{269}$ los Principios de Abiyán ofrecen a los Estados una guía para resolver las potenciales tensiones entre las obligaciones del Estado relativas a garantizar la provisión de educación pública, gratuita y de calidad

culo 10 de la Convención sobre la Eliminación de Todas las Formas de Discriminación contra la Mujer; arts. 28 y 29 de la Convención sobre los Derechos del Niño; artículo 13 del Protocolo de San Salvador; artículo 2 del Protocolo 1 de la Convención Europea de Derechos Humanos; artículo 17 de la Carta Social Europea; artículo 11 de la Carta Africana sobre los Derechos y el Bienestar del Niño y artículo 12 del Protocolo a la Carta Africana de Derechos Humanos.

267 Objetivo No. 4, Objetivos de Desarrollo Sostenible (ODS), Agenda 2030.

268 Principios de Abiyán, 2019.

269 Ibíd., Introduction. 
para todos sin discriminación, y la libertad de elegir y establecer una escuela privada, las que se producen tras la aplicación del marco de derechos humanos a la participación privada en la educación. ${ }^{270}$

Para ello, los Principios brindan a los Estados una forma de abordar estas tensiones y ofrecen puntos de referencia claros para resolverlas, basados en la reafirmación de las obligaciones estatales de respetar, proteger y cumplir el derecho a la educación. ${ }^{271}$ En concreto, este instrumento propone una serie de medidas efectivas para asegurar el derecho a la educación cuando actores privados están involucrados en su provisión, entre las que se incluyen medidas regulatorias, de supervisión y de rendición de cuentas, junto con su aplicación extraterritorial. De este modo, los Principios de Abiyán ofrecen valiosas pautas para rediseñar la organización de los sistemas educativos de manera respetuosa con los estándares internacionales de derechos humanos.

La utilidad de estos principios ha sido reconocida expresamente por varios órganos internacionales y regionales de derechos humanos con posterioridad a su adopción. ${ }^{272}$ Entre ellos se encuentran, por ejemplo, el Consejo de Derechos Humanos de Naciones Unidas ${ }^{273}$ y la Comisión Africana de Derechos Humanos y de los Pueblos. ${ }^{274}$ Asimismo, los Principios han sido destacados por organismos de monitoreo de derechos humanos, incluyendo al Relator Especial sobre Derecho a la Educación de Naciones Unidas y la Relatora Especial para los Derechos Económicos, Sociales, Culturales y Ambientales de la Comisión Interamericana de Derechos Humanos. Esta última, incluso manifestó que este instrumento es "una valiosa fuente especializada para la interpretación de la cuestión en el marco del sistema interamericano", ${ }^{275}$ al justificar su apoyo a los Principios.

Estas tensiones que los Principios de Abiyán buscan resolver no son extrañas al sistema educativo chileno. Más bien, éstas se encuentran presentes hace décadas en la discusión pública ${ }^{276}$ y tienen su origen fundamental en el marco constitucional.
C.

\section{Las tensiones del} contexto chileno
270 Ibíd., Introduction.

271 Ibíd., Introduction.

272 Lista completa de suscriptores disponible en: https://www.abidjanprinciples.org/en/support/official-recognition.

273 Consejo de Derechos Humanos de Naciones Unidas, 2019.

274 Comisión Africana de Derechos Humanos y de los Pueblos, 2019.

275 Relatoría Especial para los Derechos Económicos, Sociales, Culturales y Ambientales de la Comisión Interamericana de Derechos Humanos, 2019.

276 Ramos, 2020. 
Las políticas de mercado en materia educativa implementadas en dictadura y mantenidas en democracia, fijaron una comprensión de la educación como un bien de consumo, lo que actualmente constituye una barrera estructural para la garantía de la educación como un derecho humano. ${ }^{277}$ La profundización de esta barrera, reflejada en un modelo neoliberal que entrega su provisión al mercado, fue posible gracias a una re-conceptualización de la libertad de enseñanza como una forma de libertad de emprendimiento de los privados que obligó a entregar un tratamiento igualitario ${ }^{278}$ entre ambos sistemas (público y privado) y resultó en un abandono de la educación pública. ${ }^{279}$ Este problema ha sido subrayado tanto por el Relator Especial sobre el Derecho a la Educación en su informe sobre Chile, ${ }^{280}$ como por el Comité de Derechos Económicos, Sociales y Culturales ${ }^{281}$ y el Comité de los Derechos del Niño de las Naciones unidas. ${ }^{282}$

Estas tensiones entre lo eminentemente público y la privatización encuentran su raíz en la normativa neoliberal consagrada en la Constitución de 1980, pues a pesar de que la Carta Fundamental consagra diversos componentes del derecho a la educación en su artículo 19 Nos. 10 y 11, el texto no posee un enfoque de derechos humanos ${ }^{283}$ en la medida que garantiza únicamente el acceso a la educación sin incluir los demás elementos que configuran el derecho. ${ }^{284}$

En efecto, la actual Constitución posee una serie de falencias a la luz del enfoque de derechos:285 (i) antepone la libertad de enseñanza al derecho a la educación; (ii) no garantiza el acceso a la justicia en caso de vulneración; (iii) no contempla mecanismos de participación y gobernanza; (iv) no protege a las personas contra actos discriminatorios; (v) no garantiza la calidad de la provisión educativa; (vi) no asegura la debida adaptabilidad de la educación al territorio, identidades y necesidades estudiantiles; ni (vii) asegura un proceso formativo a lo largo de toda la vida. Estos son los nudos críticos del derecho a la educación en Chile que los Principios de Abiyán pueden ayudar a resolver.

Salgado, 2015, p. 77.

27 Falabella e llabaca, 2020.

279 Más información en: OCDE, 2012. OCDE, 2016. Bellei, 2020. Ramírez, 2021. Foro por el derecho a la educación pública, 2019, p. 5.

${ }^{280}$ Consejo de Derechos Humanos, 2017.

${ }^{281}$ Comité de Derechos Económicos, Sociales y Culturales, 2015, pár. 30.

282 Comité de Derechos del Niño, 2015, pár. 67-68 y 69-70.

283 Consejo de Derechos Humanos de Naciones Unidas, 2016, pár. 25

284 Comité de Derechos Económicos, Sociales y Culturales, 1999, p. 3.

285 González, 2020. 
De acuerdo al derecho internacional de los derechos humanos, ${ }^{286}$ los Estados se encuentran obligados a incorporar los derechos humanos en el derecho doméstico a través de todas las medidas apropiadas, las que pueden ser de carácter legislativo, administrativo, presupuestario, judicial o de cualquier otra índole. Dentro de ellas, el marco constitucional ofrece la más alta protección de estos derechos en el orden jurídico.

Tanto el Comité de Derechos Económicos, Sociales y Culturales como el ex Relator Especial sobre Extrema Pobreza y Derechos Humanos ${ }^{287}$ han resaltado la obligación de Chile de reconocer integralmente y otorgar la protección jurídica necesaria a los DESCA en el nuevo texto constitucional. ${ }^{288}$ Más aún, el reconocimiento del derecho a la educación como un derecho humano es parte fundamental de los compromisos que el Estado de Chile ha asumido desde la ratificación del Pacto Internacional de Derechos Económicos, Sociales y Culturales (PIDESC) en 1972, en un ambiente mundial donde la educación es uno de los derechos sociales con mayor protección constitucional. ${ }^{289}$

En este contexto, los Principios de Abiyán ofrecen un punto de referencia para el desarrollo de un nuevo cuadro constitucional para el derecho a la educacion en Chile, en tanto son un texto juridico que compila los estandres existentes sobre el derecho a la educacion, incluyendo las obligaciones vinculantes de los Estados. ${ }^{290}$ Por esto, son una herramienta útil para asegurar que la nueva Constitución de Chile esté alineada con las más recientes interpretaciones autorizadas sobre el derecho a la educación.

En este orden de ideas, cabe señalar que los Principios poseen una base normativa cimentada en disposiciones del derecho internacional de los derechos humanos que el Estado de Chile está obligado a respetar, proteger y cumplir. ${ }^{291}$ Adicionalmente, existen una serie de factores que justifican la legitimidad de este instrumento frente al Estado, entre los que se pueden mencionar la independencia y expertise de los redactores y signatarios; la diversidad de voces recogidas, su rigor y persuasión, su practicidad y el proceso de validación con el que fueron construidos. ${ }^{292}$ Por último, sustantivamente, y atendida la generalidad de las provisiones del derecho inter-
D.

Incorporación

normativa del derecho a la educación a la luz de los Principios de Abiyán

\footnotetext{
286 Por ejemplo, Artículo 2(1) del Pacto Internacional de Derechos Económicos, Sociales y Culturales.

287 Consejo de Derechos Humanos, 2016, pár. 71

288 Comité de Derechos Económicos, Sociales y Culturales, 2015, pár. 7.

289 Jung et al., 2014, p. 1043.

290 Op. Cit., Principios de Abiyán, p. 13

291 Sepúlveda, 2021, p. 27.

292 Ibid, pp. 33-40.
} 
nacional, la construcción específica de los Principios de Abiyán puede ser útil en la práctica para crear conciencia sobre las violaciones del derecho a la educación, identificar lagunas normativas, informar el diseño e implementación de políticas públicas en materia educativa y ofrecer directrices para que las personas directamente afectadas por violaciones al derecho a la educación busquen reparación, a la vez que pueden ser una herramienta esencial para la rendición de cuentas por parte de los Estados. ${ }^{293}$

Sin embargo y atendido el estado político y social específico de Chile, los Principios de Abiyán adquieren una importancia adicional: pueden ser una herramienta de suma utilidad para guiar las discusiones constitucionales en el marco del proceso constituyente y para lograr transformaciones profundas en el sistema educativo chileno a través de su incorporación normativa. ${ }^{294}$

$\boldsymbol{E}$.

Alineando la Constitución con el derecho a la educación
Situados en un escenario mundial donde la privatización de la educación ha crecido de manera explosiva y ha aprovechado las limitaciones de los gobiernos para hacer frente a las crecientes demandas, ${ }^{295}$ los Principios de Abiyán pueden facilitar el abordaje de diversas brechas existentes en torno al derecho a la educación en el contexto del proceso de creación de una nueva Constitución para Chile y dar dirección para el desarrollo de nuevas políticas educativas con enfoque de derechos humanos en diversos ámbitos que exploramos a continuación.

\section{i. Definir el propósito de la educación de manera más comprensiva}

El texto constitucional vigente considera que la "educación tiene por objeto el pleno desarrollo de la persona en las distintas etapas de su vida". ${ }^{296} \mathrm{Si}$ bien esta formulación avanzó respecto de las provisiones de constituciones chilenas anteriores que ponían al Estado -y no la persona- al centro de la actividad educativa, ${ }^{297}$ su redacción podría ser mejorada respecto del propó-

293 Ibid, pp. 29-30.

Op. Cit., Principios de Abiyán, principios generales $N^{\circ} 7$ y 10 y rectores 80 , 91, 92 y 95.

295 Mowbray, 2021, p. 52.

296 Constitución Política de la República de Chile, 1980, artículo 19 № 10.

297 Al respecto ver: Constitución Política del Estado, 1925, artículo 105. Constitución Política de la República de Chile, 1833, artículos 119, 144 y 145. Constitución Política de la República de Chile, 1828, artículos 114 y 122. Constitución Política de la República de Chile, 1823, artículos 38(4), 58 (5), 220(3), 254, 255, 256, 257 y 261. Constitución Política del Estado de Chile, 1822, artículos 47(19) y 230-234. 
sito enunciado por los tratados internacionales y reflejado en los Principios de Abiyán, los que consideran un doble rol de la educación: por una parte, el pleno desarrollo de la personalidad humana y el sentido de su dignidad, y por otro, la contribución al logro del bien público y a la construcción y mantención de sociedades saludables, abiertas, transparentes, tolerantes, justas, no discriminatorias e inclusivas que brinden un entorno propicio para la realización de los derechos humanos. ${ }^{298}$

La adopción de un marco comprensivo como éste para justificar el porqué de la educación, tiene la capacidad y la potencia de inspirar todas las regulaciones subsecuentes en torno a objetivo común: el desarrollo de la persona, y a través de ella, también el de su comunidad.

Cabe señalar que a nivel global formulaciones de este tipo no son extrañas, sino que se pueden encontrar en países con tradiciones tan diversas como Cuba, ${ }^{299}$ Ecuador, ${ }^{300}$ España, ${ }^{301}$ Grecia, ${ }^{302}$ Islandia, ${ }^{303}$ México, ${ }^{304}$ Noruega, ${ }^{305}$ y Portugal. ${ }^{306}$

\section{Constitución de Islandia}

Artículo 24. (...) La educación tendrá por objeto lograr el desarrollo integral de cada individuo, el pensamiento crítico y la conciencia de los derechos humanos, los derechos y las obligaciones democráticas.

\section{ii. Garantizar la disponibilidad de una educación pública, gratuita,} inclusiva y de buena calidad para todos

Los Principios de Abiyán ayudan a clarificar la naturaleza y el alcance del derecho a una educación pública. ${ }^{307} \mathrm{Si}$ bien el Pacto reconoce la garantía del acceso universal y la gratuidad progresiva de los diversos niveles de enseñanza, cuya responsabilidad hace radicar en los Estados, no alude expresamente a un sistema de educación pública, a pesar de existir innumerables indicios en el derecho internacional que consagran la obligación de proveer

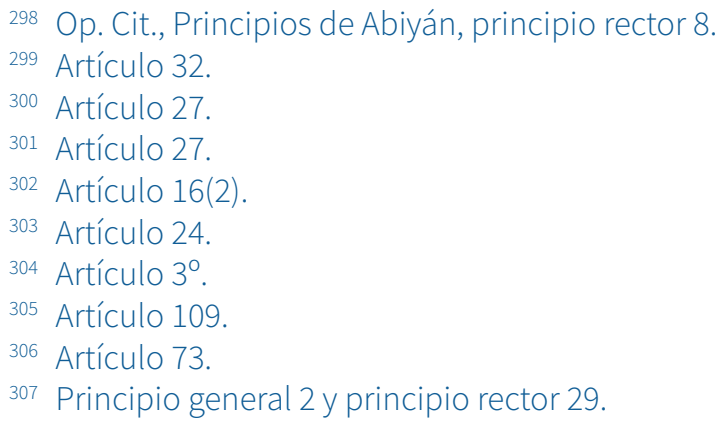


este tipo de educación, ${ }^{308}$ protegiéndose otras formas como una excepción, una adición o un suplemento a la educación pública. ${ }^{309}$

Los Principios de Abiyán realizan una lectura contextualizada que destaca esta ausencia, reivindica las motivaciones tras la adopción del Pacto y reformula la obligación de los Estados a través de su principio general 2, que en lo sustantivo señala que:

Los Estados deben proporcionar educación pública, gratuita de la más alta calidad posible a todas las personas dentro de su jurisdicción de la manera más eficaz y rápida posible, utilizando el máximo de sus recursos disponibles.

Más aún, en otros numerales, los Principios de Abiyán proporcionan otros elementos sustantivos para revelar lo que en el Pacto parece ausente: la cualidad de servicio público de la educación. ${ }^{310}$ En este sentido, los Principios someten la provisión de la educación a las garantías propias del suministro de servicios públicos, obligaciones que alcanzan incluso a las instituciones educativas privadas. El cambio es profundo: al conceptualizarse como servicio público, "el Estado deja de limitar su actuación a la preservación de la libertad y a la garantía de los derechos individuales - propiedad privada y libertad de comercio- y se convierte en protagonista directo del crecimiento económico y del progreso social". ${ }^{311}$

Si bien el texto de la Constitución actual considera la obligación del Estado de promover y garantizar la educación parvularia, básica y media, la estrategia utilizada para traducir este mandato se reduce únicamente al deber de financiar un sistema gratuito, ${ }^{312}$ obviando cualquier consideración que requiera organizar la actividad estatal en torno a un verdadero servicio público de educación bajo un marco respetuoso de los derechos humanos, es decir, un servicio accesible y no discriminatorio, responsivo a la necesidades de sus usuarios y culturalmente apropiado, participativo, de calidad suficiente, adecuadamente financiado, transparente y responsable. ${ }^{313}$

Algunos países del mundo han constitucionalizado la idea de la educa-

Op. Cit., Mowbray, 2021, p. 57.

Ibíd., p. 56.

310 Op. Cit., Principios de Abiyán, numeral 19. Otros elementos también pueden encontrarse en los numerales 11, 12, 13, 31, 37 y 39.

311 Troncoso, 1999, p. 98.

312 Constitución Política de la República de Chile, 1980, artículo 19 № 10.

313 The Global Initiative for Economic, Social and Cultural Rights, 2020, pp. 3 y 4. 
ción pública como principio organizador de la sociedad, entre los que se pueden identificar a Costa de Marfil, ${ }^{114}$ Costa Rica, ${ }^{315}$ Dinamarca, ${ }^{316}$ Ecuador, ${ }^{317}$ Francia, ${ }^{318}$ Holanda, ${ }^{319}$ México, ${ }^{320}$ Portugal ${ }^{321}$ y Suiza. ${ }^{322}$

\section{Constitución de Costa de Marfil}

Artículo 10. (...) El Estado garantiza la promoción y el desarrollo de la educación pública general, la educación técnica y la formación profesional, así como la expansión de todos los sectores, de acuerdo con las normas internacionales de calidad y en relación con las necesidades del mercado laboral. (...)

\section{iii. Limitación de la libertad de enseñanza y regulación de los ac- tores privados en la educación}

La libertad de enseñanza, incluyendo la libertad de elección de la escuela y la libertad de establecer y dirigir escuelas, tal cual se encuentra establecida en los tratados de derechos humanos, no es absoluta.

El espíritu original que existía detrás de esta libertad era permitir que los padres impidieran que sus hijos fueran condicionados por un estado tiránico y permitir que los padres con creencias fuertes o necesidades lingüísticas educaran a sus hijos conforme a ellas. ${ }^{323}$ Sin embargo, esta disposición ha sido sostenidamente reinterpretada y cooptada por la promoción de la mercantilización y el retroceso del Estado, a través de la instalación del proyecto neoliberal. ${ }^{324}$ Bajo este paradigma, la Constitución chilena establece de manera prioritaria "el derecho de abrir, organizar y mantener establecimientos educacionales" sin otras "limitaciones que las impuestas por la moral, las buenas costumbres, el orden público y la seguridad nacional"; asegurando residualmente el derecho de los padres de "escoger el establecimiento de enseñanza para sus hijos". Bajo esta lógica, cualquier intento regulatorio se observa como una intervención estatal que busca expropiar

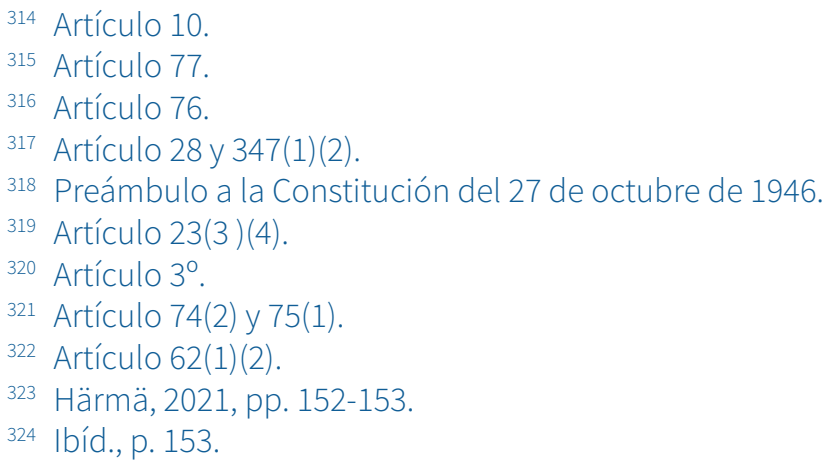


las expectativas de ganancia. ${ }^{325}$

Así, se evidencia que un fuerte énfasis en la elección convierte a los ciudadanos en consumidores que buscan el mejor "trato" para sus hijos, en lugar de miembros de un colectivo que apoyan una escuela que pertenece a toda la comunidad. ${ }^{326}$ A su vez, la conceptualización de este derecho como absoluto, produce el efecto perverso de que su ejercicio a menudo disminuye materialmente los derechos y los resultados de los niños menos afortunados. ${ }^{327}$

En este sentido, el principio rector 48 de los Principios de Abiyán recuerda que estas libertades no son absolutas y que el derecho internacional de los derechos humanos impone una serie de límites específicos y concretos al ejercicio de estas libertades, entre los que se relevan: la no suplantación o reemplazo de la educación pública y la no creación de impactos sistémicos adversos sobre el derecho a la educación, los que incluyen la generación o mantenimiento de disparidades de oportunidades o resultados para algunos grupos de la sociedad y la afectación de la capacidad del Estado para realizar el derecho a una educación pública, gratuita y de calidad.

En esta materia, el proceso constituyente ofrece la oportunidad a Chile para adecuar la garantía constitucional de la libertad de enseñanza a los estándares de derechos humanos, incluyendo al aseguramiento de que la existencia de actores privados no conduzca a la comercialización de la educación ${ }^{328}$ y que en caso de financiarse la educación privada con fondos públicos, se resguarde siempre la garantía del derecho a la educación sin discriminación . ${ }^{329}$

\section{iv. Garantizar la buena gobernanza del sistema educativo publico}

En materia de gobernanza, el aporte de los Principios de Abiyán es sustantivo: ${ }^{330}$ sus provisiones estipulan que los Estados deben aplicar los principios de dignidad humana, participación, igualdad y no discriminación, inclusión, transparencia y rendición de cuentas en la prestación y la gobernanza del servicio público de educación. ${ }^{331}$ Más aún, consagran la obligación específica de establecer un sistema de gobernanza educativa participativa que sea representativa de todas las partes interesadas, incluidos los

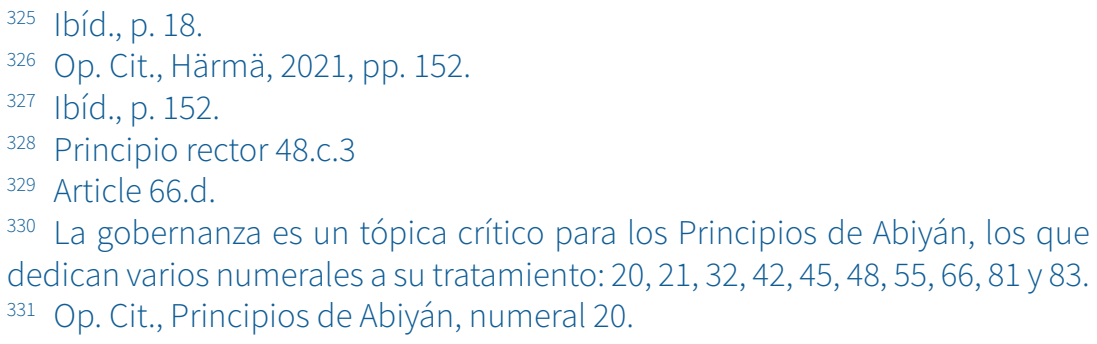


niños y otros estudiantes, los padres o tutores legales, las comunidades, el personal docente y no docente, los sindicatos de la educación y otras organizaciones de la sociedad civil. ${ }^{332}$

En esta materia la brecha es profunda, en la medida que la Constitución chilena se limita a establecer tímidamente un deber de la comunidad de "contribuir al desarrollo y perfeccionamiento de la educación",333 sin establecer forma alguna de cumplir este deber. En contraste, constituciones como las de Cuba, ${ }^{334}$ Ecuador, ${ }^{335}$ España,, ${ }^{336}$ Grecia, ${ }^{337}$ Indonesia,, ${ }^{338}$ Mé$x^{x i c 0^{339}}$ y Portugal ${ }^{340}$, contemplan diversos mecanismos de participación de las comunidades educativas en los sistemas de educación.

\section{Constitución de Portugal}

Artículo 77: Participación democrática en la educación

1. Los profesores y los estudiantes tendrán derecho a participar en la gestión democrática de las escuelas, según lo establece la ley.

2. La ley regulará las formas en las que las asociaciones de profesores, alumnos y padres, las comunidades y las instituciones científicas participaran en la elaboración de la política educativa.

\section{v. Establecer los principios de igualdad y no discriminación}

Los Principios de Abiyán también entregan propuestas concretas para consolidar la igualdad y no discriminación en la educación. Para ello recuerdan, por ejemplo, la obligación de los Estados de adoptar todas las medidas necesarias para eliminar todas las formas de discriminación en el disfrute del derecho a la educación por motivos prohibidos, que incluyan tanto la discriminación directa e indirecta, el acoso y la negación de ajustes razonables, y la discriminación múltiple, interseccional, asociativa y perceptiva. ${ }^{341}$ Asimismo, se incluye el enfoque de género al requerir que los

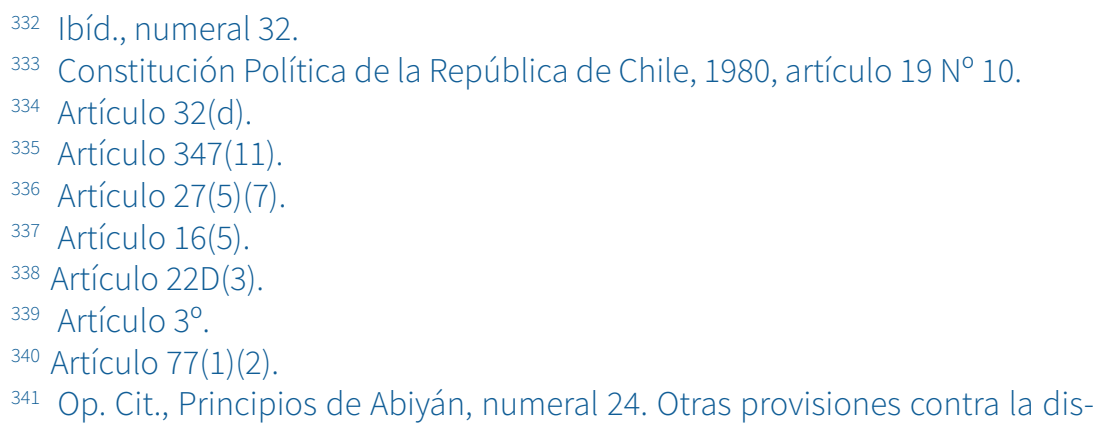


Estados diseñen y adopten una estrategia nacional de educación sensible al género, a las necesidades de las niñas y las mujeres y transformadora para todos. ${ }^{342}$

Ambas estrategias propuestas por los Principios de Abiyán pueden ser útiles para una próxima formulación del derecho a la educación en Chile, pues la Constitución actual carece de provisiones específicas que prohíban la discriminación por cualquier motivo en la educación ${ }^{343}$ y que incorporen el enfoque de género con especial énfasis en una educación no sexista como una herramienta de cambio social.

A nivel global, numerosas constituciones han adoptado diversas tácticas para garantizar la igualdad, inclusión y no discriminación en la educación. Dentro de ellas se encuentran las cartas fundamentales de Corea del Sur, ${ }^{344}$ Costa de Marfil, ${ }^{345}$ Cuba, ${ }^{346}$ Ecuador, ${ }^{347}$ Eslovaquia, ${ }^{348}$ Eslovenia, ${ }^{349}$ España, ${ }^{350}$ Finlandia, ${ }^{351}$ Hungría, ${ }^{352}$ Italia, ${ }^{353}$ Japón, ${ }^{354}$ México, ${ }^{355}$ Noruega, ${ }^{356}$ Portugal, ${ }^{357}$ Singapur ${ }^{358}$ y Turquía, ${ }^{359}$ Más aún, algunas de ellas incorporan provisiones específicas que abordan la igualdad de género, tales como las constituciones de Costa de Marfil ${ }^{360}$, Ecuador ${ }^{361}$ y Polonia. ${ }^{362}$

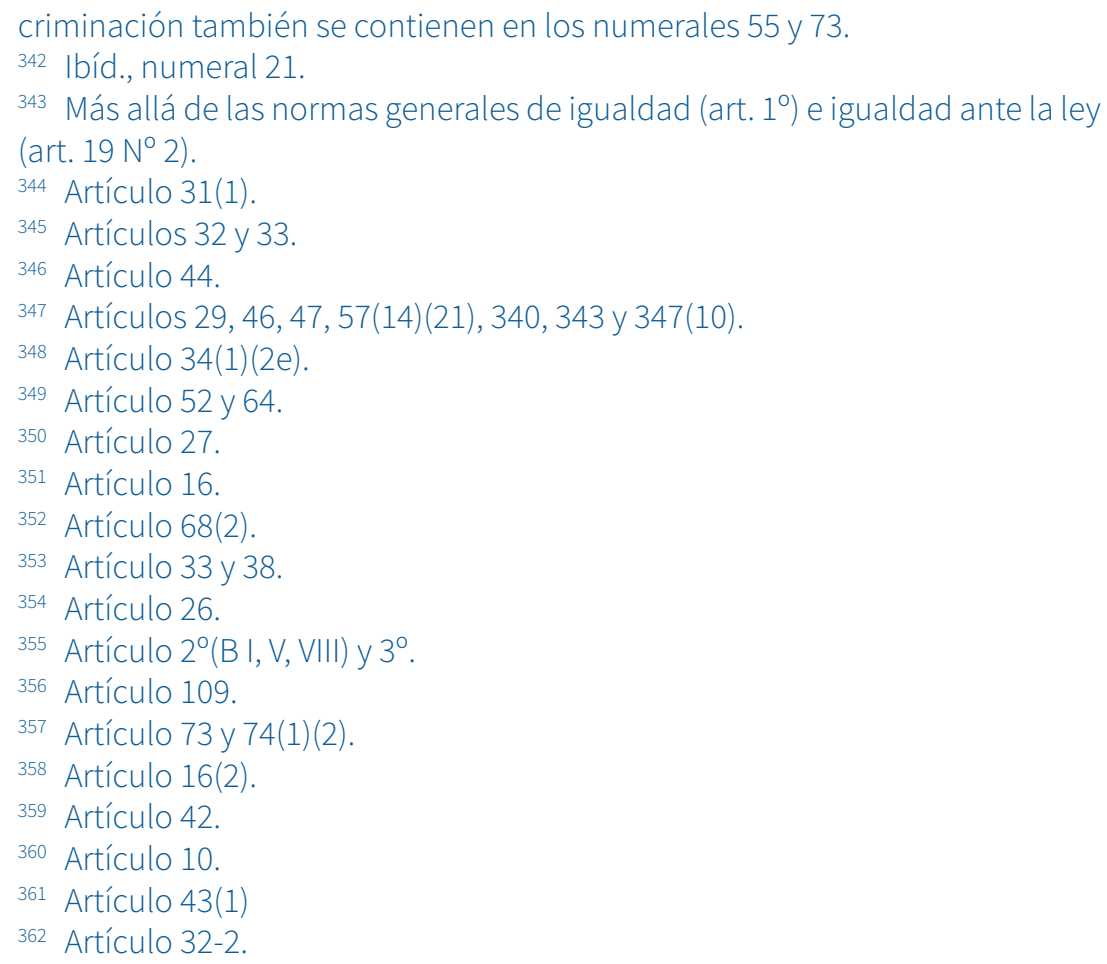




\section{Constitución de Polonia}

Artículo 32-2. Hombres y mujeres tendrán iguales derechos, en particular, respecto a la educación, (...)

\section{vi. Garantizar el financiamiento adecuado de la educación publica}

Los Principios de Abiyán desarrollan la importancia fundamental del financiamiento de la educación, ${ }^{363}$ poniendo énfasis en la necesidad de dar prioridad al financiamiento de la educación publica como también en la exigencia de financiar instituciones educativas privadas únicamente cuando se observen estrictamente una serie de requisitos establecidos a nivel sustantivo, procedimental y operativo. ${ }^{364}$ Los Principios de Abiyán recuerdan que los Estados deben proporcionar educación pública, gratuita, de la más alta calidad posible a todas las personas que se encuentren bajo su jurisdicción de la manera más eficaz y expedita posible, utilizando el máximo de sus recursos disponibles. ${ }^{365}$ Precisan además que los recursos máximos disponibles no deben caer por debajo del nivel requerido por los compromisos nacionales o internacionales de financiación de la educación, como el porcentaje del PIB establecido en sus objetivos de desarrollo. ${ }^{366}$

Las declaraciones internacionales, tales como la Declaración de Jomtien de 2011, reconocen que los Estados deben asignar por lo menos el $6 \%$ de su PIB y/o al menos un 20\% de sus presupuestos nacionales a educación para alcanzar una educación de calidad para todos y todas. ${ }^{367}$ Es así que constituciones como las de Costa Rica ${ }^{368}$ e Indonesia ${ }^{369}$ ya garantizan el presupuesto nacional de educación dentro de su texto fundamental. En el proceso de elaboración de la nueva Constitución, Chile podría asegurar un nivel adecuado de financiamiento para la educación publica incluyendo alguna provisión relacionada en materia educativa.

\footnotetext{
Ver sección IV. Financiamento; Principio general 5 y principio rector 64.

364 Principios rectores 63 a 74.

365 Principio general 2.

366 Principio rector 15.

367 La Declaración de Incheon de 2015 recomienda que los Estados asignen por lo menos entre un $4 \%$ y un $6 \%$ del Producto Interno Bruto y/o al menos entre un 15\% y un 20\% del gasto público total en educación (Educación 2030, 2016, p. 9).

368 Artículo 78

369 Artículo 31(4).
} 
Articulo 78, párrafo 2. "En la educación estatal, incluida la superior, el gasto publico no será inferior a seis por ciento (6\%) anual del producto interno bruto, de acuerdo con la ley (...)"

\section{vii. Reconocer la justiciabilidad del derecho a la educación}

Por último, como medida de clausura, los Principios de Abiyán requieren que los Estados garanticen la disponibilidad de mecanismos de reclamación y reparación rápidos, accesibles, eficaces, procesalmente justos e independientes -incluidos cuando sea necesario, los recursos judiciales-, que permitan a cualquier titular de derechos o a otras partes interesadas, como los grupos de interés público, estar equipados con la capacidad de reclamar derechos y buscar reparación por cualquier abuso de derechos humanos por parte de un actor involucrado en la educación. ${ }^{370}$ Este aporte puede subsanar una profunda brecha en relación con la Constitución chilena, que en la actualidad sólo permite hacer exigible a través de la acción de protección la libertad de enseñanza en los términos establecidos anteriormente. 371

Si bien a nivel comparado los mecanismos de exigibilidad varían en su naturaleza, constituciones como las de Argentina, ${ }^{372}$ Colombia $^{373}$ y Estonia ${ }^{374}$ consideran el derecho a la educación como un derecho justiciable.

\section{Constitución de Colombia}

Artículo 86. Toda persona tendrá acción de tutela para reclamar ante los jueces, en todo momento y lugar, mediante un procedimiento preferente y sumario, por si misma o por quien actúe a su nombre, la protección inmediata de sus derechos constitucionales fundamentales, cuando quiera que éstos resulten vulnerados o amenazados por la acción o la omisión de cualquier autoridad pública. (...)

\footnotetext{
370 Op. Cit., Principios de Abiyán, numeral 89. También se consagra esta circunstancia en el numeral 80.

${ }^{371}$ Constitución Política de la República de Chile, 1980, artículo 20.

372 Artículo 43.

373 Artículo 86.

374 Artículo 15.
} 
Hace poco más de 2 años, los Principios de Abiyán fueron adoptados por un grupo amplio y diverso de expertos de derechos humanos a través de un proceso legítimo, para abordar los desafíos globales de la privatización y mercantilización de la educación. Respecto de estos procesos Chile ha representado un escenario prolífico: la Constitución de 1980 y las reformas educativas basadas en los mecanismos del mercado han jugado un rol fundamental en la comprensión de la educación como un bien privado, convirtiendo el sistema educativo de este país en uno de los más privatizados y segregados del mundo.

Frente a este escenario, y de acuerdo al derecho internacional de los derechos humanos, Chile se encuentra obligado a incorporar el derecho a la educación dentro de su marco normativo interno, siendo la Constitución la norma suprema. En este contexto de incorporación doméstica, los Principios de Abiyán se posicionan como un instrumento jurídicamente relevante para guiar la discusión constitucional en relación al derecho a la educación, al poner especial énfasis en elementos constitucionales que resultan claves para la implementación de un sistema educacional basado en los principios de justicia social.

Este ensayo ha explorado la forma en que los Principios de Abiyán pueden resultar de utilidad para resolver las tensiones existentes respecto del derecho a la educación, en puntos críticos como la primacía de la educación pública por sobre la libertad de enseñanza; el establecimiento de medidas de igualdad sustantiva y no discriminación; la inclusión de mecanismos de gobernanza que fomenten la participación de la comunidad en materia educativa, la garantía de un financiamiento adecuado y la necesidad de establecer mecanismos judiciales y de rendición de cuentas para que los Estados puedan remediar las violaciones del derecho humano a la educación.

De este modo, es posible concluir que la constitucionalización de los Principios de Abiyán en Chile permitiría reconocer y garantizar de manera irrestricta el derecho a la educación pública, gratuita y de calidad para todas y todos, incluyendo todas sus dimensiones y su función social. Esta opción por la incorporación doméstica de los Principios podría facilitar, en el largo plazo, el cumplimiento de las obligaciones internacionales vigentes desde hace décadas en Chile, transformando desde su base normativa un sistema educativo históricamente marcado por la segregación y desigualdad.
F.

Conclusión 
Bellei, C. et al. (2020)

'La fragilidad de la escuela pandémica', CIPER, 19 diciembre. Disponible en: https://www.ciperchile.cl/2020/12/19/la-fragilidad-de-la-escuela-pandemica/ (Consulta: 6 junio 2021).

Comisión Africana de Derechos Humanos y de los Pueblos (2019)

Resolución sobre la obligación de los Estados de regular a los agentes privados que participan en la prestación de servicios de salud y educación. Documento no. 420 (LXIV).

Comité de Derechos Económicos, Sociales y Culturales (1990)

Observación General N³. La índoles de las obligaciones de los Estados Partes (párrafo 1 del artículo 2 del Pacto). Disponible en: https:// tbinternet.ohchr.org/_layouts/15/treatybodyexternal/Download.aspx?symbolno=INT\%2fCESCR\%2fGEC\%2f4758\&Lang=en (Consulta: 6 junio 2021).

Comité de Derechos Económicos, Sociales y Culturales (1999)

Observaciones generales 13 (21ํ período desesiones, 1999) El derecho a la educación (artículo 13 del Pacto). Documento No. E/C.12/1999/10. Disponible en: https://undocs.org/es/E/C.12/1999/10 (Consulta: 6 junio 2021).

Comité de Derechos Económicos, Sociales y Culturales (2015)

Observaciones finales sobre el cuarto informe periódico de Chile. Documento No. E/C.12/CHL/CO/4. Disponible en: https://undocs.org/ es/E/C.12/CHL/CO/4 (Consulta: 6 junio 2021).

Comité de Derechos Económicos, Sociales y Culturales de Naciones Unidas (1999)

Observación General N 13. El derecho a la educación. Documento No. E/C.12/1999/10. Disponible en: https://tbinternet.ohchr.org/_ layouts/15/treatybodyexternal/Download.aspx?symbolno=E\%2fC.12\%2f1999\%2f10\&Lang=en (Consulta: 6 junio 2021).

Comité de Derechos Económicos, Sociales y Culturales de Naciones Unidas (2015)

Observaciones finales sobre el cuarto informe periódico de Chile. Documento No. E/C.12/CHL/CO/4. Disponible en: https://undocs.org/ es/E/C.12/CHL/CO/4 (Consulta: 16 junio 2021). 
Comité de los Derechos del Niño de Naciones Unidas (2001)

Observación General No 1: párrafo 1 del artículo 29: propósitos de la educación. Documento No. CRC/GC/2001/1. Disponible en: https:// undocs.org/es/CRC/GC/2001/1 (Consulta: 6 junio 2021).

Comité de los Derechos del Niño de Naciones Unidas (2015)

Observaciones finales sobre los informes periódicos cuarto y quinto combinados de Chile. Documento No. CRC/C/CHL/CO/4-5. Disponible en: https://tbinternet.ohchr.org/_layouts/15/treatybodyexternal/ Download.aspx?symbolno=CRC/C/CHL/CO/4-5\&Lang=Sp (Consulta: 16 junio 2021).

Consejo de Derechos Humanos de Naciones Unidas (2016) Informe del Relator Especial sobre la extrema pobreza y los derechos humanos sobre su misión a Chile. Documento No. A/HRC/32/31/ Add.1. Disponible en: https://documents-dds-ny.un.org/doc/UNDOC/GEN/G16/072/51/PDF/G1607251.pdf?OpenElement (Consulta: 6 junio 2021).

Consejo de Derechos Humanos de Naciones Unidas (2017)

Informe del Relator Especial sobre el derecho a la educación sobre su misión a Chile. Documento No. A/HRC/35/24/Add.1. Disponible en: https://documents-dds-ny.un.org/doc/UNDOC/GEN/G17/081/48/ PDF/G1708148.pdf?OpenElement (Consulta: 26 junio 2021).

Consejo de Derechos Humanos de Naciones Unidas (2019)

El derecho a la educación: el ejercicio efectivo del derecho a la educación y la consecución del Objetivo de Desarrollo Sostenible 4 en el contexto del aumento de las entidades del sector privado en el ámbito de la educación. Documento No. A/HRC/41/37. Disponible en: https://undocs.org/es/A/HRC/41/37 (Consulta: 6 junio 2021).

Educación 2030:

Declaración de Incheon y Marco de Acción para la realización del Objetivo de Desarrollo Sostenible 4: Garantizar une educación inclusiva y equitativa de calidad y promover oportunidades de aprendizaje permanente para todos (2016) Documento No. ED-2016/WS/28. Disponible en: https://unesdoc.unesco.org/ark:/48223/pf0000245656_ spa (Consulta: 16 junio 2021).

Falabella, A. e llabaca, T. (2020)

'Cómo terminar con el lugar privilegiado de la educación privada en Chile, CIPER, 21 de diciembre. Disponible en: https://www.ciperchile. cl/2020/12/26/como-terminar-con-el-lugar-privilegiado-de-la-educacion-privada-en-chile/ (Consulta: 6 junio 2021).

Foro por el derecho a la educación pública (2019)

Informe luz de la situación de la educación en Chile al 2019. Disponible en: http://derechoeducacionpublica.cl/wp-content/ 
uploads/2019/07/FODEP-2019-Informe-Luz-de-la-Situaci\%C3\%B3nde-la-Educaci\%C3\%B3n-en-Chile.pdf (Consulta: 6 junio 2021).

González, J. et al. (2020)

Constituyamos Otra Educación, Una Mejor Sociedad Es Posible: 10 nudos constitucionales en educación y experiencias internacionales destacadas para inspirar el debate nacional. Disponible en: https:// www.summaedu.org/wp-content/uploads/2020/10/CONSTITUYENTE-DOC_FINAL_PAG.pdf (Consulta: 6 junio 2020).

Härmä, J. (2021)

'Evidencie on school choice and the human right to education, en Adamson, F. et al., Realizing the Abidjan Principles on the right to education. Human Rights, Public Education and the Role of Private Actors in Education. UK; USA: Edward Elgar Publishing Limited. DOI: $10.4337 / 9781839106033$.

Jung, C. et al. (2014)

'Economic and social rights in national constitutions', American Journal of Comparative Law, 62(4).

Mowbray, J. (2021)

'Is there a right to public education?', en Adamson, F. et al., Realizing the Abidjan Principles on the right to education. Human Rights, Public Education and the Role of Private Actors in Education. UK; USA: Edward Elgar Publishing Limited. DOI: 10.4337/9781839106033.

\section{Principios de Abiyán:}

Principios rectores sobre las obligaciones de derechos humanos que incumben a los Estados de proporcionar educación pública y regular la participación del sector privado en la educación (2019) Disponible en: https://www.abidjanprinciples.org/es/home (Consulta: 11 junio 2021).

Ramírez, N. (2021)

'Alarmante impacto de la pandemia: Estudiantes de $6^{\circ}$ básico a III medio no alcanzaron el 60\% de los aprendizajes en 2020', EMOL, 26 mayo. Disponible en: https://www.emol.com/noticias/Nacional/2021/05/26/1021974/Alarmante-resultado-diagnostico-estudiantes.html (Consulta: 6 junio 2021)

Ramos, M. (2020)

'Hemos llegado a un punto en que el éxito de los colegios privados está produciendo un daño al sistema público de educación', CIPER, 5 abril. Disponible en: https://www.ciperchile.cl/2020/04/05/hemosllegado-a-un-punto-en-que-el-exito-de-los-colegios-privados-estaproduciendo-un-dano-al-sistema-publico-de-educacion/ (Consulta: 6 junio 2021). 
Red ONGs Infancia y Juventud Chile (2019)

Lista de Cuestiones Previa a la Presentación de Informes (List of issues prior to Reporting) a presentar ante el Comité de Derechos del Niño en el marco de la 88ava sesión. Disponible en: https://tbinternet.ohchr.org/Treaties/CRC/Shared\%20Documents/CHL/INT_CRC_ NGO_CHL_40848_S.pdf (Consulta: 29 mayo 2021).

Relatoría Especial para los Derechos Económicos, Sociales, Culturales y Ambientales de la Comisión Interamericana de Derechos Humanos (2019)

Empresas y Derechos Humanos: Estándares Interamericanos. Disponible en: https://www.oas.org/es/cidh/informes/pdfs/EmpresasDDHH.pdf (Consulta: 6 junio 2021).

Salgado, C. (2015)

'El derecho a la educación', en Bassa, J. et al. (ed.) La Constitución chilena. Una perspectiva crítica a su práctica política. Santiago: LOM Editores.

Sepúlveda, M. (2021)

'Human Rights Guiding Principles: A forward-looking retrospective', en Adamson, F. et al., Realizing the Abidjan Principles on the right to education. Human Rights, Public Education and the Role of Private Actors in Education. UK; USA: Edward Elgar Publishing Limited. DOI: 10.4337/9781839106033.

The Global Initiative for Economic, Social and Cultural Rights (2020)

States' Human Rights Obligations Regarding Public Services The United Nations Normative Framework. Disponible en: https://static1. squarespace.com/static/5a6e0958f6576ebde0e78c18/t/5fc6235e3f75b16643a5bd59/1606820703234/2020-10-19-Policy-Brief-States-HR-Oblig-PS-UN-NormFram.pdf (Consulta: 6 junio 2021).

Tomasevski, K. (2009)

Human rights obligations: making education available, accessible, acceptable and adaptable. CLACSO. Disponible en: http://bibliotecavirtual.clacso.org.ar/Argentina/lpp/20100426090811/11.pdf (Consulta: 6 junio 2021).

Troncoso, A. (1999)

'Dogmática Administrativa y Derecho Constitucional: el caso del servicio público'. Revista Española De Derecho Constitucional, 57. Disponible en: http://www.jstor.org/stable/24883723 (Consulta: 6 junio 2021). 